\title{
Full counting statistics of stationary particle beams
}

Cite as: J. Math. Phys. 54, 042109 (2013); https://doi.org/10.1063/1.4801780

Submitted: 19 June 2012 . Accepted: 27 March 2013 . Published Online: 23 April 2013

J. Kiukas, A. Ruschhaupt, and R. F. Werner

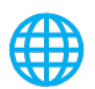

View Online

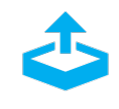

Export Citation

\section{ARTICLES YOU MAY BE INTERESTED IN}

Measurement uncertainty relations

Journal of Mathematical Physics 55, 042111 (2014); https://doi.org/10.1063/1.4871444

Screen observables in relativistic and nonrelativistic quantum mechanics

Journal of Mathematical Physics 27, 793 (1986); https://doi.org/10.1063/1.527184

Bell's inequalities and quantum field theory. I. General setting

Journal of Mathematical Physics 28, 2440 (1987); https://doi.org/10.1063/1.527733

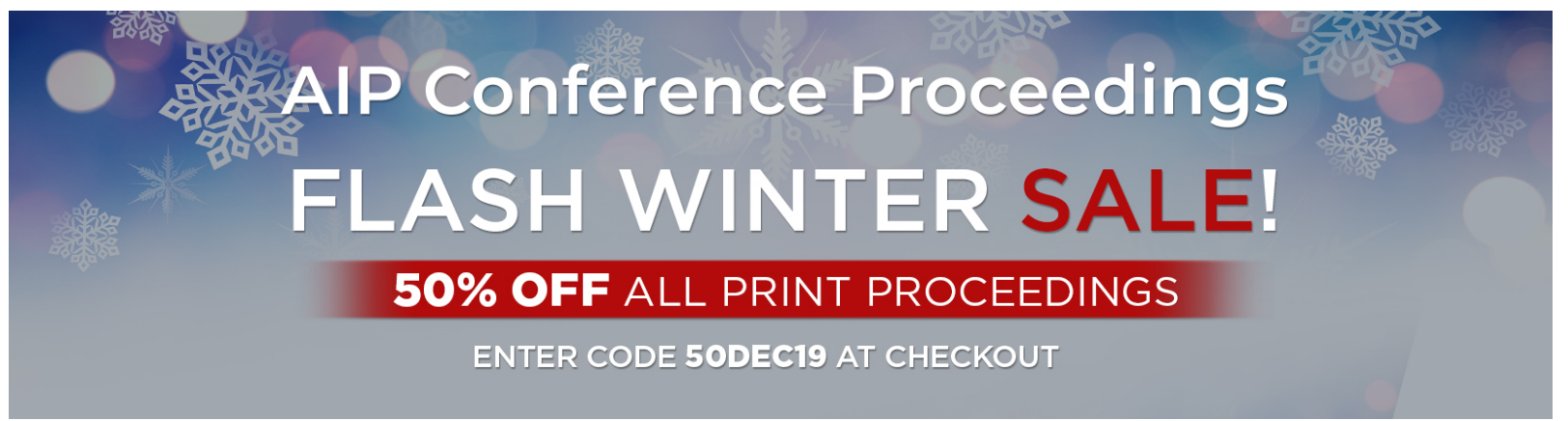




\title{
Full counting statistics of stationary particle beams
}

\author{
J. Kiukas, ${ }^{1}$ A. Ruschhaupt, ${ }^{2}$ and R. F. Werner ${ }^{3}$ \\ ${ }^{1}$ Zentrum Mathematik, M5, Technische Universität München, Boltzmannstrasse 3, \\ 85748 Garching, Germany \\ ${ }^{2}$ Department of Physics, University College Cork, Cork, Ireland \\ ${ }^{3}$ Inst. Theoret. Physik, Leibniz Universität Hannover, Appelstr. 2, 30167 Hannover, Germany
}

(Received 19 June 2012; accepted 27 March 2013; published online 23 April 2013)

We present a general theoretical framework for treating particle beams as timestationary limits of many particle systems. Due to stationarity, the total particle number diverges, and a description in Fock space is no longer possible. Nevertheless, we show that when describing the particle detection via second quantized arrival time observables, such beams exhibit a well-defined "local" counting statistics, that is, full counting statistics of all clicks falling into any given finite time interval. We also treat in detail a realization of such a beam via the long time limit of a source creating particles in a fixed initial state from which they then evolve freely. From the mathematical point of view, the beam is described by a quasi-free state which, in the one-particle level, is locally trace class with respect to the operator valued measure describing the time observable; this ensures the existence of a Fredholm determinant defining the characteristic function of the counting statistics. (C) 2013 AIP Publishing LLC. [http://dx.doi.org/10.1063/1.4801780]

\section{INTRODUCTION}

The statistical interpretation of quantum mechanics asserts that all predictions of the theory are about probabilities estimated as relative frequencies in repeated measurements. In each "single shot" of such a run of repeated experiments just one quantum system will be prepared and then measured on. However, this scenario is rarely realized in real experiments: It is much more common to prepare a stream of systems by some source, and to use detectors, which are continuously active and may click whenever a particle triggers them in the right way. The final result of such an experiment is then not a probability but a count rate, normalized to the production rate of the source. It is obvious that these two pictures (see Fig. 1) are closely related, and often the normalized count rates are simply identified with probabilities.

While the theoretical concepts needed to account for repeated single particle experiments are well established, the tools for describing beams, especially stationary beams, are not so well developed. It is the aim of this paper to close this gap, and to provide a clear conceptual and mathematical framework for beams. We believe that this may help to clarify some issues like the controversy about the correct quantum state describing a propagating laser beam. ${ }^{1-3}$ By focusing on the full time resolved counting statistics it may also help to give a systematic background to timing issues in cryptography ${ }^{4}$ or foundational experiments. ${ }^{5}$ We were led to this problem in the course of a project on the tomography of single photon sources. Clearly, in this case it is relatively easy to get beam-type data, in which, for example, the antibunching dip in the $g^{(2)}$-correlation function indicates single particle emission. ${ }^{6,7}$ On the other hand, the emission time of individual particles is not controlled in beam mode, so absolute click times are meaningless. Hence it is impossible to get a full tomography of the single particle state created by such a source on demand; beam data and single-particle statistics have to be combined. But this requires a coherent structure in which both make sense.

The comparison of mathematical structures for single-particle theory and theory of beams is summarized in Table I. As an intermediate column we added many-particle systems, which also 
(a)

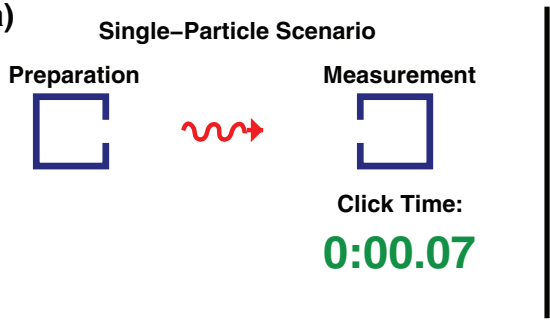

(b)

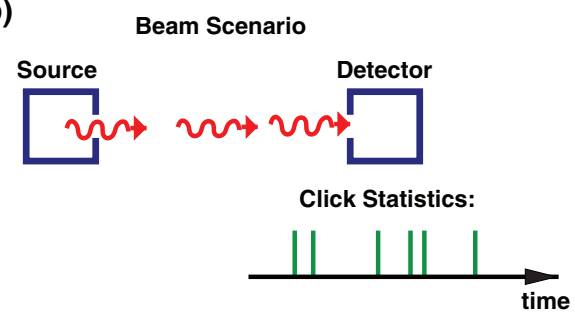

FIG. 1. Schematical comparison between (a) the single-particle scenario and (b) the beam scenario (right).

reflects our construction procedure. From the single-particle description one gets to this level by the formalism of "Second Quantization." This is then adequate for beams of finite duration, which have a finite total number of particles. Implicitly, at this level one is forced to describe the initial and final transients, which are, however, irrelevant for the practical description of stationary beams. The purpose of this paper is to go to the third column, the stationary limit, in which one gets a simplified description without transients.

In the standard framework of quantum mechanics each type of systems is assigned a Hilbert space $\mathcal{H}$, and a Hamiltonian $H$. This setting will be kept on all levels. For the many-particle systems this becomes Fock space with the corresponding second quantized Hamiltonian of non-interacting systems. The statistical character of the systems (Bosons or Fermions), which is irrelevant at the single particle level, plays a crucial role here. The Hilbert space of the infinite beam is not explicitly used, because we carry out the stationary limit on the level of expectation values. It could be constructed as the Gelfand-Naimark-Segal (GNS) Hilbert space of the stationary state in a certain quantum field theory over the time axis, but for the present paper we found no advantage in introducing these structures. The key element of our construction are arrival time observables, which are covariant with respect to the given time evolution. In second quantization this becomes an observable whose output space consists of click sequences or, more formally, counting measures, which assign to any time interval the number of clicks in that interval. The expectation of such an observable in any state is thus a point process. ${ }^{8}$ Of course, its statistical properties depend on the state. Here we make a very special assumption, which we do intend to lift in future work, namely, that the beam particles are as uncorrelated as possible under the Bose/Fermi constraint. Technically this means that we consider quasi-free states. This assumption is very natural if not canonical, when we look at beam experiments as analogs of single-shot measurements: The successive shots are assumed to be independent in any estimation of probabilities, so quasi-freeness is the appropriate formulation of this standing assumption in the beam setting. One could go even further and require that the beam is so thin that the statistical character of the particles becomes irrelevant, but we found this unnecessary.

On the other hand there are many interesting beams which do not satisfy this independence assumption, and for which the inter-particle correlations contain the relevant information sought by

TABLE I. Comparison of mathematical structures for three settings described in the text.

\begin{tabular}{|c|c|c|c|}
\hline & Single particle & Many particles & Beam \\
\hline Hilbert space & $\mathcal{H}$ & Fock space over $\mathcal{H}$ & Not explicitly used \\
\hline State & Density operator & Quasi-free density operator & Non-normal quasi-free state \\
\hline Measurement & Arrival time POVM & \multicolumn{2}{|c|}{ 2nd quantized arrival time POVM } \\
\hline \multirow[t]{2}{*}{ Outcome } & Click time & \multirow{2}{*}{\multicolumn{2}{|c|}{ Sequence of click times }} \\
\hline & & & \\
\hline Statistics & & Global & Local \\
\hline 1st moment & Expectation & \multicolumn{2}{|c|}{ Count rate } \\
\hline 2nd moment & Variance & \multicolumn{2}{|c|}{ Correlation functions } \\
\hline
\end{tabular}


the experiment. A typical example is the light from continuously driven "single photon sources." Here the successive photons are correlated because the emitting atom or vacancy center needs time to recover after each emission. There are several formalisms for dealing with such correlations, such as the cavity input-output formalism, ${ }^{6}$ quantum stochastic integration, ${ }^{9}$ or a continuous time version of matrix-product states. ${ }^{10,11}$ In these approaches, however, the emitted particles usually do not undergo their own intrinsic time evolution, which creates some problems in combining these approaches with the framework of the current paper, which is explicitly built on the time evolution of the beam particles. We intend to resolve these problems in the future.

Our paper is organized as follows. In Sec. II we describe the detection side of the scheme, i.e., arrival time observables and their second quantization. In order to make the paper reasonably self-contained, we begin in with brief statements of some relevant prerequisites from the theory of counting processes. ${ }^{8,12,13}$ In Sec. III we come to the preparation side, describing the class of quasi-free states in Fock space, and compute the full counting statistics for such states. Prerequisites comprise Fredholm determinants, ${ }^{14}$ which are needed to give an explicit description of the full counting statistics. Point processes rather similar to the ones we find have been discussed in the mathematical literature under the heading of "determinantal processes." ${ }^{15}$ However, to the best of our knowledge, these were always considered to be stationary in space ${ }^{16,17}$ rather than in time, and so do not involve the machinery of arrival time positive operator valued measures (POVMs) needed in our case. Section IV contains the main result of the paper: we extend the counting statistics for quasi-free states to the time-local counting statistics of stationary beams. In particular, Sec. IV B is devoted to a concrete way of going to the stationary limit starting from an explicit dynamical description of particle creation. In Secs. IV C and IV D we give a general scheme for describing local counting statistics for quasi-free stationary beams; in particular, we get explicit formulas for correlation functions. Finally, Sec. V is devoted to some examples: We apply the results to compute the second order correlation function for a quasi-free beam stationary for the free Hamiltonian, measured using the simplest time-covariant observable. We also look at plane waves viewed as many-particle states. Often enough such an interpretation is suggested in textbook treatments of scattering solutions of the stationary Schrödinger equation for one-particle potential scattering. Here we take it literally, and as a bonus get correlations and waiting time distributions in such a beam.

\section{COUNTING OBSERVABLES}

Let us fix some notation. Throughout, $\mathcal{H}$ will be the Hilbert space of a single particle. By $\Gamma_{s}(\mathcal{H})$ we denote the Fock space over $\mathcal{H}$, with $s=+1$ for Bosons and $s=-1$ for Fermions. When it is irrelevant, or clear from the context, the index $s$ will be omitted. For an operator $A$ on $\mathcal{H}$, we will denote by $\Gamma_{s}(A)$ the operator, which on $N$-particle wave functions acts like the $N$-fold tensor power $A^{\otimes N}$. Clearly, $\Gamma_{s}(A B)=\Gamma_{s}(A) \Gamma_{s}(B)$.

For operator valued or scalar valued measures $\mu$ we abbreviate the integral over a scalar function $f$ as

$$
\mu[f]=\int \mu(d x) f(x),
$$

i.e., we use round brackets for the set function and square brackets for the integral, i.e., the expectation value functional in the case of a probability measure.

For observables (POVMs = "positive operator valued measures") the discrete case, in which points have finite measure, is often used. Then $F[f]=\sum_{x} f(x) F_{x}$, where $F_{x}$ are positive operators with $F[1]=\sum_{x} F_{x} \leq \mathbb{1}$. The projection valued special case is characterized by $F_{x} F_{y}=\delta_{x y} F_{x}$, or in a form also valid in the continuous case: $F[f g]=F[f] F[g]$.

In most textbooks, observables are simply identified with self-adjoint operators $A$, which presupposes that $X \subset \mathbb{R}$ and takes $F$ as the spectral measure of $A$, so $A=\int F(d x) x=F[x]$. The same measure also defines the one-parameter unitary group $\exp (i t A)=F\left[e^{i t x}\right]$ generated by $A$. Generators are second quantized by $A \mapsto \mathrm{d} \Gamma(A)$, where $\Gamma(\exp (i t A))=\exp (i t \mathrm{~d} \Gamma(A))$ for all $t$. However, for the purpose of this paper it is much more appropriate to start from POVMs on some outcome space $X$, which need not be the real line. The point is that for the natural second quantization of such 
$\mathrm{POVMs}^{18}$ the outcome space also changes: where the observable $F$ at the one-particle level gives the probability of outcomes $x \in X$, its second quantization $\Gamma F$ will correspond to a measurement of $F$ on every particle, and the result of this measurement is a distribution of points in $X$.

The probabilities for such measurement outcomes constitute a so-called "point process." In the following we will first describe the relevant facts about such processes; for a detailed theory, we refer to Ref. 8. In this framework, we will then formulate the structure of second quantized arrival time POVMs, which provide abstract description for the measurement of detection times for a system of quantum particles.

\section{A. Point processes}

A point process is a probability measure on the outcome space consisting of collections of (not necessarily distinct) points in a set $X$. We can think of each outcome as a possibly infinite numbered list $(x(1), x(2), \ldots)$ with $x(i) \in X$, with the understanding that the ordering of the elements is irrelevant but, in contrast to the set $\{x(1), x(2), \ldots\}$, we do count the number of occurrences of each $x \in X$. A way to express this compactly is to take as the outcome of a point process the so-called empirical measure

$$
\xi=\sum_{i} \delta_{x(i)},
$$

where $\delta_{x}$ denotes the point measure (with $\delta$-function density) at $x \in X$. A measure $\xi$ of this form is also called a counting measure, and is characterized among measures by the property that the measure of each set is an integer, namely, the number of points $x_{i}$ in that set. Using the bracket notation introduced above, we then have $\xi[f]=\sum_{i} f(x(i))$ for an empirical measure $\xi=\sum_{i} \delta_{x(i)}$. Since this bracket is linear in $f$, we can use it to characterize the probability distribution of a point process by its Fourier transform, i.e., by the expectation $\langle\cdot\rangle$ of the function $\xi \mapsto \exp (i \xi[f])$. This is called the characteristic function

$$
C(f)=\left\langle e^{i \xi[f]}\right\rangle
$$

of the distribution and contains the full counting statistics.

As an example, let us consider the finite case $|X|<\infty$. Then for a large total number of outcomes each point $x \in X$ occurs several times. The typical random variables to describe the system are thus the occupation numbers $n(x)=|\{i \mid x(i)=x\}|$, which are just the weights of the empirical measure $\xi=\sum_{x} n(x) \delta_{x}$. Consider the occupation number tuples $\mathbb{N}^{X}$ as $|X|$-tuples as tuples of real numbers. Then the point process amounts to a probability distribution on $\mathbb{R}^{X}$ concentrated on integer points. Its Fourier transform, i.e., the characteristic function in the usual sense, is the expectation of $\exp (\mathrm{in} \cdot f)$ $=\exp \left(i \sum_{x} n(x) f(x)\right)=\exp (i \xi[f])$, where in the first expression the dot indicates the scalar product in $\mathbb{R}^{X}$. The values of the function $f(x)$ are thus the components of the vector valued Fourier variable. This finite setting arises frequently from the continuous case by coarse graining, i.e., when we ask reduced questions. Consider a partition of $X$ into $k$ disjoint subsets $X_{\ell}$ of $X$, and let $\chi_{\ell}$ denote the indicator function of $X_{\ell}$. If we want to evaluate the joint distribution $p\left(n_{1}, \ldots, n_{k}\right)$ of occupation numbers $n_{\ell}=\left|\left\{i \mid x(i) \in X_{\ell}\right\}\right|$, it suffices to evaluate the characteristic function on the subalgebra of step functions $f(x)=\sum_{\ell=1}^{k} f_{\ell} \chi_{\ell}(x)$. The $f_{\ell}$ are then the components of the Fourier vector, i.e., we get the desired joint distribution from

$$
\sum_{n_{1}, \ldots n_{k}} p\left(n_{1}, \ldots n_{k}\right) e^{i \sum_{\ell} f_{\ell} n_{\ell}}=\left\langle e^{i \sum_{\ell} f_{\ell} \xi\left(X_{\ell}\right)}\right\rangle=C\left(\sum_{\ell} f_{\ell} \chi_{\ell}\right)=C(f)
$$

If the particle numbers are independent for every partition of $X$ into sets $X_{\ell}$, we have a Poisson process, which is characterized by a measure $\mu$ on $X$, called the intensity measure of the process, such that $p\left(n_{1}, \ldots n_{k}\right)=\prod_{\ell} \mu\left(X_{\ell}\right)^{n} /(n !) \exp \left(-\mu\left(X_{\ell}\right)\right.$ and hence,

$$
C(f)=\exp \int \mu(d x)\left(e^{i f}-1\right) .
$$


The $k$ th moment of the point process is defined as the uniquely determined permutation symmetric measure $m_{k}$ on $X^{k}$, satisfying

$$
\int m_{k}\left(d x_{1} \cdots d x_{k}\right) \prod_{j=1}^{k} f\left(x_{j}\right)=\left\langle\xi[f]^{k}\right\rangle .
$$

Using arbitrary functions $f$ on $X^{k}$, we can equivalently give the definition as

$$
m_{k}[f]=\left\langle\sum_{i_{1}, \cdots, i_{k}} f\left(x\left(i_{1}\right), \ldots, x\left(i_{k}\right)\right)\right\rangle .
$$

Since

$$
C(f)=\sum_{k} \frac{i^{k}}{k !} \int m_{k}\left(d x_{1} \cdots d x_{k}\right) \prod_{j=1}^{k} f\left(x_{j}\right),
$$

we can extract the moments from $C(\lambda f)$ by differentiating with respect to $\lambda$. For a Poisson process, the expansion of the characteristic function in powers of $f$ is $C(f)=i \mu[f]-\frac{1}{2}\left(\mu\left[f^{2}\right]+\mu[f]^{2}\right)$ $+\mathbf{O}\left(f^{3}\right)$, so the first moment is $m_{1}=\mu$ and the second is $m_{2}=\mu \otimes \mu+\mu \circ \Delta^{-1}$ with the diagonal map $\Delta(x)=(x, x)$. The second moment thus has a singular part concentrated on the diagonal. This is not a special feature of the Poisson process, but occurs for any counting process. It is, therefore, customary to consider a modified set of moments, called factorial moments, ${ }^{8}$ or "correlation functions," 15 which do not have such singularities. Like the moment $m_{k}$, the factorial moment of order $k$, which we denote by $\widehat{m}_{k}$, is a permutation symmetric measure on $X^{k}$. For a function $f$ of $k$ variables, the factorial moment is defined by the following expectation:

$$
\widehat{m}_{k}[f]=\left\langle\sum_{\substack{i_{1}, \cdots i_{k} \\ \text { distinct }}} f\left(x\left(i_{1}\right), \ldots, x\left(i_{k}\right)\right)\right\rangle .
$$

By comparing the expression (7) to (5) it is clear that the exclusion of multiply occurring indices in the former just has the effect of eliminating the singular term from the second moment. For the Poisson process one has $\widehat{m}_{k}=\mu^{\otimes k}$ for all $k$.

The factorial moments are most easily obtained from the characteristic function $C$ by observing that the generating function

$$
\widehat{C}(f)=\sum_{k} \frac{1}{k !} \int \widehat{m}_{k}\left(d x_{1} \cdots d x_{k}\right) \prod_{j=1}^{k} f\left(x_{j}\right)
$$

is related to $C$ just by a transformation of the argument:

$$
C(f)=\widehat{C}\left(e^{i f}-1\right) .
$$

As an example of using (9), consider the probability $p_{Y}(n)$ of finding exactly $n$ particles in a measurable region $Y \subset X$. By (3) and (9), we get the relation

$$
\sum_{n=0}^{\infty} p_{Y}(n) z^{n}=\widehat{C}\left((z-1) \chi_{Y}\right)
$$

for $z=e^{i \lambda}$, where $\chi_{Y}$ is the indicator function of $Y$. In particular, we get the no event probability directly from the factorial moment generating function by analytic continuation:

$$
p_{Y}(0)=\widehat{C}\left(-\chi_{Y}\right) \text {. }
$$

These probabilities determine the interval statistics of a point process on the time axis $X=\mathbb{R}$. Indeed, let $p_{0}\left(t_{1}, t_{2}\right)=p_{\left[t_{1}, t_{2}\right)}(0)$ denote the probability for not finding a click in the interval $\left[t_{1}, t_{2}\right)$. Then the probability of having no click on $\left[t_{1}, t_{2}\right)$ and at least one click just before $t_{1}$, say in the interval $\left[t_{1}-\varepsilon, t_{1}\right)$, is the same as having no click on $\left[t_{1}, t_{2}\right)$ and at least one in $\left[t_{1}-\varepsilon, t_{2}\right)$, which 
is $p_{0}\left(t_{1}, t_{2}\right)-p_{0}\left(t_{1}-\varepsilon, t_{2}\right)$. Hence the conditional probability for having no click on $\left[t_{1}, t_{2}\right)$, on the condition of having at least one in $\left[t_{1}-\varepsilon, t_{1}\right)$, is

$$
\frac{p_{0}\left(t_{1}, t_{2}\right)-p_{0}\left(t_{1}-\varepsilon, t_{2}\right)}{1-p_{0}\left(t_{1}-\varepsilon, t_{1}\right)} .
$$

At the limit $\varepsilon \rightarrow 0$, this tends to $1-$ the probability of having to wait at most time $\tau=t_{2}-t_{1}$ for the next click, after a click at $t_{1}$. Hence the probability density $w_{s}(\tau)$ for the waiting time $\tau \in[0, \infty)$ given a click at $s$ is

$$
w_{s}(\tau)=-\left(\frac{\partial p_{0}}{\partial t_{1}}(s, s)\right)^{-1} \frac{\partial^{2} p_{0}}{\partial t_{1} \partial t_{2}}(s, s+\tau)
$$

Since we will eventually apply the above formalism to particle detection processes, we close this subsection by a remark on the role of the factorial moment densities in the standard theory of photon counting used in quantum optics. If $X$ has a natural measure $d x$ (typically $X=\mathbb{R}^{m}$ with the Lebesgue measure), we can often write $\widehat{m}_{k}\left(d x_{1} \ldots d x_{k}\right)=h^{(k)}\left(x_{1}, \ldots, x_{k}\right) d x_{1} \ldots d x_{k}$ for a density function $h^{(k)}$. In Glauber's model of a photon detection process, we have

$$
h^{(n)}\left(x_{1}, \ldots, x_{n}\right)=\mathrm{const} \cdot G^{(n)}\left(x_{1}, \ldots, x_{n}, x_{n}, \ldots, x_{1}\right),
$$

where $G^{(n)}$ is the usual "correlation function" defined using the field operators. ${ }^{6,19}$ In this context, one also typically uses the normalized correlation functions, which we define for a general point process by

$$
g^{(n)}\left(x_{1}, \ldots, x_{n}\right)=\frac{h^{(n)}\left(x_{1}, \ldots, x_{n}\right)}{h^{(1)}\left(x_{1}\right) \cdots h^{(1)}\left(x_{n}\right)}
$$

\section{B. Second quantization of general observables}

One reason for introducing characteristic functions of point processes is that they make the construction of the second quantized observable $\Gamma F$ from the single particle observable $F$ extremely simple. ${ }^{18}$ Indeed, if we just express the idea that $\Gamma F$ measures $F$ on all the particles we get, restricted to the $N$-particle space, the operator

$$
\left((\Gamma F)\left[e^{i \xi[f]}\right]\right)_{N}=\int F\left(d x_{1}\right) \otimes \cdots \otimes F\left(d x_{N}\right) e^{\sum_{i} f\left(x_{i}\right)}=\left(F\left[e^{i f}\right]\right)^{\otimes N} .
$$

Taking the direct sum over $N$, we get the fundamental formula

$$
(\Gamma F)\left[e^{i \xi[f]}\right]=\Gamma\left(F\left[e^{i f}\right]\right) .
$$

This expression makes sense on full Fock space, i.e., it does not require restriction to the Bose or Fermi sector. Hence, for a state given by a density operator $\rho$ on this space the full counting statistics can be extracted from the characteristic function

$$
C(f)=\operatorname{tr} \rho(\Gamma F)\left[e^{i \xi[f]}\right]=\operatorname{tr} \rho \Gamma\left(F\left[e^{i f}\right]\right) .
$$

Of course, for a Bose or Fermi system, the operator $\rho$ has support in the appropriate subspace and we can replace the $\Gamma$ in this formula by the corresponding restriction $\Gamma_{s}$.

The factorial moments can be computed from this using Eqs. (9) and (8). For a state $\rho$ on full Fock space we get

$$
\widehat{C}(f)=\operatorname{tr} \rho \Gamma(\mathbb{1}+F[f])=\sum_{N=0}^{\infty} \operatorname{tr} \rho_{N}(\mathbb{1}+F[f])^{\otimes N}=\sum_{k=0}^{\infty} \operatorname{tr} \hat{\rho}_{k} F[f]^{\otimes k},
$$

where $\hat{\rho}_{k}$ is the reduced $k$-particle reduced density operator

$$
\hat{\rho}_{k}=\sum_{N=k}^{\infty}\left(\begin{array}{l}
N \\
k
\end{array}\right) \operatorname{tr}_{[k+1, \ldots, N]} \rho_{N} .
$$


Hence the factorial moments are

$$
\widehat{m}_{k}\left(d x_{1} \cdots d x_{k}\right)=k ! \operatorname{tr} \hat{\rho}_{k} F\left(d x_{1}\right) \otimes \cdots F\left(d x_{k}\right) .
$$

\section{Arrival time observables and their dilations}

Due to an old argument of Pauli, an arrival time observable cannot be a spectral measure of a self-adjoint "time operator." However, the generalization of the notion of observables to POVMs immediately allows time-shift covariant observables to be constructed..$^{20}$ In this subsection we describe the general construction of observables, which measure the arrival time $t$ and arrival location $x$ of a particle. ${ }^{21}$ Here location is taken in a rather broad sense, and could just be the number of the detector which responds. We consider arbitrary observables, which are covariant for time translations, i.e.,

$$
\exp (i H t) F[f] \exp (-i H t)=F\left[\tau_{t} f\right]
$$

where $H$ is the Hamiltonian, and $\tau$ is the time shift on functions of $t$ and $x$, i.e., $\left(\tau_{t} f\right)\left(x, t^{\prime}\right)$ $=f\left(x, t^{\prime}-t\right)$.

As an example consider a free particle on the line, i.e., $\mathcal{H}=\mathcal{L}^{2}(\mathbb{R}, d x)$, with Hamiltonian $H \psi$ $=-\left(\hbar^{2} / 2 m\right) \psi^{\prime \prime}$. Diagonalizing $H$ is to take the Fourier transform and rewriting the Hilbert space in the variables $E=p^{2} / 2 m$ and $\eta=\operatorname{sign} p$, so we get $\mathcal{H} \cong \mathcal{L}^{2}\left(\mathbb{R}_{+}, d E\right) \otimes \mathbb{C}^{2}$ with Hamiltonian $H \psi(E$, $\eta)=E \psi(E, \eta)$. In this representation a "time operator" would be $\frac{i}{\hbar} \frac{d}{d E}$. However, this operator is not self-adjoint, since it has one non-zero defect index arising from the boundary condition at zero. This is just a reflection of Pauli's argument. However, we can consider the larger Hilbert space $\widetilde{\mathcal{H}}=\mathcal{L}^{2}(\mathbb{R}, d E) \otimes \mathbb{C}^{2}$, i.e., we formally allow also negative energies. In this extended space the time operator is a well-defined self-adjoint operator canonically conjugated to the multiplication operator "energy." We can use it to compute probability distributions of time in the usual way, with the only modification that the states we consider all lie in the "physical subspace" with positive energy. This gives the Kijowski distributions, ${ }^{20}$ which can be written as $\operatorname{tr} \rho F(\cdot)$ for a POVM $F$ satisfying (19).

This example generalizes to a much wider setting. The standard method ${ }^{21}$ to build all covariant observables (even for a general covariance group with representation $g \mapsto U_{g}$ ) involves two steps: one first uses the Naimark dilation theorem to turn any generalized (POVM) observable into a projection valued one, say $\widetilde{F}$, which lives on another Hilbert space $\widetilde{\mathcal{H}}$ and is connected to $F$ by an isometry $V: \mathcal{H} \rightarrow \widetilde{\mathcal{H}}$ so that $F[f]=V^{*} \widetilde{F}[f] V$. There is also a unitary group representation $\widetilde{U}$ on $\widetilde{\mathcal{H}}$, for which $\widetilde{F}$ is covariant, and which is intertwined by $V$, i.e., $\widetilde{U}_{g} V=V U_{g}$. In the second step one uses the theory of Mackey ${ }^{22}$ who called projection valued covariant observables "systems of imprimitivity" and showed their intimate connection to induced representations. This second part is easy for just the time translation group $\mathbb{R}$, and leads to standard Schrödinger pairs of position and momentum operators, with some multiplicity. Thus in the dilation space "energy" is the multiplication operator canonically conjugated to "time," and has therefore purely absolutely continuous spectrum. Since it is connected by an intertwining isometry $V$ with the given Hamiltonian the covariant time observable approach is limited to Hamiltonians $H$ with absolutely continuous spectrum. Absolutely continuous spectrum will therefore be our standing assumption on the Hamiltonian in the sequel. Diagonalizing it leads to a representation of the Hilbert space in direct integral form

$$
\mathcal{H}=\int^{\oplus} d E \mathcal{H}_{E} .
$$

This is shorthand for the space of wave functions, which are functions of energy such that $\psi(E)$ $\in \mathcal{H}_{E}$, the multiplicity space at $E$. This will, of course, be $\{0\}$ when $E$ is not in the spectrum of $H$ (e.g., when $E<0$ for the standard kinetic energy). Scalar products are computed as

$$
\langle\phi \mid \psi\rangle_{\mathcal{H}}=\int d E\langle\phi(E) \mid \psi(E)\rangle_{\mathcal{H}_{E}}
$$


with the scalar product of $\mathcal{H}_{E}$. The technical (measurability) conditions on direct integral Hilbert spaces are to ensure that this expression makes sense (see, e.g., Ref. 23). Of course, the Hamiltonian is the multiplication operator $(H \psi)(E)=E \psi(E)$ in this representation. More generally, a bounded operator $A$ commutes with $H$ if $(A \psi)(E)=A_{E} \psi(E)$ for some measurable family of operators $A_{E} \in \mathcal{B}\left(\mathcal{H}_{E}\right)$. We write for this

$$
A=\int^{\oplus} d E A_{E}
$$

If a space (20) allows a projection valued covariant time observable, and hence a self-adjoint conjugate time operator, this operator generates a unitary group which shifts the energy variable. It thus introduces a canonical unitary identification between all the spaces $\mathcal{H}_{E}$. In particular, they must be non-zero also for negative energies, excluding all semi-bounded Hamiltonians. Nevertheless, this structure appears as the dilation of any given time observable. The Hilbert space in that case can be written either as the tensor product $\mathcal{L}^{2}(\mathbb{R}, d E) \otimes \mathcal{K}$ or, in the spirit of (20), as the space of $\mathcal{K}$-valued $\mathcal{L}^{2}$-functions on $\mathbb{R}$. The key difference to the general case of (20) is that now $\mathcal{K}_{E}=\mathcal{K}$ for all $E$. The time observable in this case is computed in the usual way by Fourier-transforming to $\mathcal{L}^{2}(\mathbb{R}, d t) \otimes \mathcal{K}$, and the joint measurement of $t$ and $x$ is realized in this tensor product. It is thus characterized by the following data:

1. a Hilbert space $\mathcal{K}$, which will be the energy-independent multiplicity space of the dilated observable,

2. a family of isometries $V_{E}: \mathcal{H}_{E} \rightarrow \mathcal{K}$, which together define the dilation isometry $V: \mathcal{H} \rightarrow$ $\mathcal{L}^{2}(\mathbb{R}, d E) \otimes \mathcal{K}$ via $(V \psi)(E)=V_{E} \psi(E)$, and

3. an observable $G$ with outcome space $X$ in the Hilbert space $\mathcal{K}$.

We have to compute the expectation operator $F[h]$ for arbitrary functions $f$ of $(t, x)$ but it suffices to do this for the product functions $f(t, x)=h(t) g(x)$. For these the above data determine the operator

$$
F[h g] \psi(E)=\int d E^{\prime} \widehat{h}\left(E-E^{\prime}\right) V_{E}^{*} G[g] V_{E^{\prime}} \psi\left(E^{\prime}\right),
$$

where $\widehat{h}$ denotes the Fourier transform of $h$, normalized as

$$
\widehat{h}(E)=\frac{1}{2 \pi} \int d t e^{i E t} h(t) .
$$

This ensures that for $g=1$ and $h \nearrow 1$ we find $F(h) \nearrow \mathbb{1}$, so the observable $F$ is normalized. It is convenient to allow also subnormalized observables, i.e., $F(1) \leq \mathbb{1}$. In that case the operator $\mathbb{1}-F(1)$ measures the probability that the particle never arrives. By construction this operator will commute with $H$, and the only modification in the above setup is to allow $V$ to be a general operator with $\|V\| \leq 1$, rather than an isometry.

\section{QUASI-FREE STATES}

\section{A. Physical background}

Let us begin with a Boltzmann statistical model of a multi-particle preparation: Suppose we have a one-particle preparation with density operator $\sigma_{0}$, which we run at $N$ random times $t_{i}$. Hence, if $\sigma(t)=e^{-i H t} \sigma_{0} e^{i H t}$ is the time translate of $\sigma_{0}$, we get the state $\bigotimes_{i=1}^{N} \sigma\left(t_{i}\right)$. We ignore for the moment the symmetrization requirements, so we apply the observable $F$ to each of these systems separately, obtaining a point $x_{i}$ as a measuring result. In order to determine the characteristic function of the counting statistics we need the distribution of the emission times, which we take to be Poisson with intensity measure $\mu$ (i.e., with characteristic function $C_{\text {time }}(g)=\exp \int \mu(d t)\left(e^{i g}-1\right)$, see (4)). From this we get the characteristic function of the counts $x_{i}$ as

$$
C(f)=\exp \int \mu(d t) \operatorname{tr} \sigma(t) F\left(e^{i f}-1\right) .
$$


This is again Poisson, and depends only on the integral $\sigma=\int \mu(d t) \sigma(t)$. The corresponding state on full Fock space is $\bigoplus_{N} \frac{1}{N !} \sigma^{\otimes N}$, where the factorial is the usual correction factor for indistinguishability familiar in classical statistical mechanics.

Of course, this state is not consistent with Bose or Fermi statistics. Its closest analogue is to replace $\frac{1}{N !} \sigma^{\otimes N}$, by $P_{s} \sigma^{\otimes N} P_{s}$, where $P_{s}$ denotes the projection onto the (anti-)symmetric subspace of $\mathcal{H}^{\otimes N}$. That is, we consider the quasi-free state with density operator

$$
\rho=\frac{\Gamma_{s}(\sigma)}{\operatorname{tr} \Gamma_{s}(\sigma)}
$$

on $\Gamma_{s} \mathcal{H}$. Here we do not take $\sigma$ to be normalized. Instead the normalization factor of $\sigma$ determines the particle number distribution. To be precise, (26) is a "gauge invariant" quasi-free state. More general quasi-free states, which do not necessarily commute with particle number are defined in Ref. 24, and will not be studied in this paper. Quasi-free states are plausible models for noninteracting particle beams, because they give the same results as Boltzmannian independence in the weak beam limit. They also describe naturally the production of a beam. Consider an oven, modeled as an ideal gas with one-particle Hamiltonian $H$, at temperature $T=1 /(k \beta)$, and chemical potential $\mu$. Then we have the grand canonical quasi-free state with $\sigma=\exp (-\beta(H-\mu \mathbb{1}))$. We then get a beam by letting some particles escape through a hole, and we can also add (possibly time-dependent) one-particle potentials, collimating filters, and the like. The important point is that as long as we only apply one-particle operations, i.e., unitary operators of the form $\Gamma(U)$, the quasi-free character of the initial state will be preserved.

In the context of quantum optics this type of photon beam is usually called thermal light (see, e.g., Ref. 6), the state appearing as a special case of "chaotic state." 19 The latter is defined in terms of the occupation number states $\left|\left\{n_{k}\right\}\right\rangle$ as

$$
\rho=\sum_{\left\{n_{k}\right\}} \prod_{k} \frac{\alpha_{k}^{n_{k}}}{\left(1+\alpha_{k}\right)^{1+n_{k}}}\left|\left\{n_{k}\right\}\right\rangle\left\langle\left\{n_{k}\right\}\right|,
$$

where $k$ indexes the modes, and the $\alpha_{k}>0$ are parameters satisfying $\sum_{k} \alpha_{k}<\infty$, each $\alpha_{k}$ coinciding with the expectation value of the mode $k$ occupation number. In fact, any quasi-free state of the form (26) for $s=1$ can be written as (27) by choosing the modes according to an eigenbasis of the positive trace class operator $\sigma$; the parameters $\alpha_{k}$ are then the eigenvalues of $\widehat{\sigma}=\sigma /(\mathbb{1}-\sigma)$.

\section{B. Characteristic functions}

A crucial tool in the following is a formula for the denominator in (26). When $A$ is trace class (i.e., $\|A\|_{1}=\operatorname{tr} \sqrt{A^{\dagger} A}<\infty$ ), and, in the Bose case $\|A\|<1$, then $\Gamma_{s}(A)$ is also trace class and

$$
\operatorname{tr} \Gamma_{s}(A)=\operatorname{det}(\mathbb{1}-s A)^{-s} .
$$

For the theory of such infinite dimensional determinants we refer to Ref. 14. Using this formula, we get a simple expression for the characteristic function of a counting measurement:

$$
\begin{aligned}
C(f) & =\frac{\operatorname{tr} \Gamma(\sigma) \Gamma\left(F\left[e^{i f}\right]\right)}{\operatorname{tr} \Gamma(\sigma)}=\frac{\operatorname{tr} \Gamma\left(\sigma F\left[e^{i f}\right]\right)}{\operatorname{tr} \Gamma(\sigma)}=\frac{\operatorname{det}\left(\mathbb{1}-s \sigma F\left[e^{i f}\right]\right)^{-s}}{\operatorname{det}(\mathbb{1}-s \sigma)^{-s}} \\
& =\operatorname{det}\left(\mathbb{1}-s(\mathbb{1}-s \sigma)^{-s} \sigma\left(F\left[e^{i f}\right]-\mathbb{1}\right)\right)^{-s} .
\end{aligned}
$$

To summarize

$$
\begin{aligned}
C(f) & =\operatorname{det}\left(\mathbb{1}-s \widehat{\sigma} F\left[e^{i f}-1\right]\right)^{-s}, \\
\text { with } \quad \widehat{\sigma} & =\frac{\sigma}{\mathbb{1}-s \sigma},
\end{aligned}
$$

where we took the liberty to write a fraction because numerator and denominator commute, and the expression can be evaluated in the functional calculus. It is useful to note the bounds on the operators 
$\sigma, \widehat{\sigma}$ in the Bose and Fermi case: Clearly both operators must be positive and have finite trace. In the Bose case we need in addition that $\sigma \leq(1-\varepsilon) \mathbb{1}$ for some $\varepsilon>0$, which is equivalent to saying that $\widehat{\sigma}$ is bounded. In the Fermi case it is the other way around: $\sigma$ can be any bounded operator, which implies that $\widehat{\sigma}$ is strictly less than the identity. The formula (29) contains the complete counting statistics for the counting observable (compare also Ref. 25).

\section{Factorial moments}

From (29) and (9) we get the factorial moment generating function

$$
\widehat{C}(f)=\operatorname{det}(\mathbb{1}-s \widehat{\sigma} F[f])^{-s}=\operatorname{tr} \Gamma_{s}(\widehat{\sigma} F[f]) .
$$

Comparing this with (16) we see that the $k$-particle reduced density operators of the quasi-free state are $\hat{\rho}_{k}=P_{s} \widehat{\sigma}^{\otimes k} P_{s}$, so by (18), the factorial moments are given by

$$
\widehat{m}_{k}\left(d x_{1} \cdots d x_{k}\right)=k ! \operatorname{tr} P_{s} \widehat{\sigma}^{\otimes k} P_{s} F\left(d x_{1}\right) \otimes \cdots F\left(d x_{k}\right) .
$$

The first moment is simply $\widehat{m}_{1}(d x)=\operatorname{tr} \widehat{\sigma} F(d x)$; for the second moment, the expression can be further reduced, so that traces have only to be taken in the one particle space. To this end we write the (anti-)symmetrization projection $P_{s}=(\mathbb{1}+s \mathbb{F}) / 2$, where $\mathbb{F}$ is the unitary transposition operator, and use $\operatorname{tr}(\mathbb{F} A \otimes B)=\operatorname{tr}(A B)$. Then

$$
\widehat{m}_{2}(d x d y)=\widehat{m}_{1}(d x) \widehat{m}_{1}(d y)+s \operatorname{tr}(\widehat{\sigma} F(d x) \widehat{\sigma} F(d y)) .
$$

Now the trace on the right-hand side is a positive measure on $X \times X$ and is also positive definite in the sense that it gives positive expectation to functions of the form $\overline{f(x)} f(y)$. This shows that for Bosons we always have the bunching effect $g^{(2)} \geq 1$ and the antibunching effect $g^{(2)} \leq 1$ for Fermions. (Recall the definition (13) of the correlation function $g^{(2)}$.) Clearly, there are interesting cases of photon antibunching, but these require artfully correlated, not quasi-free sources.

For later use we note the $k$ th order generalization of (33). The expression for $\operatorname{tr}\left(V_{\pi} A_{1} \otimes \cdot A_{k}\right)$, for a permutation operator $V_{\pi}$ is based on the cycle decomposition of the permutation $\pi$, say $\pi=\left(i_{1}, \ldots, i_{r}\right)\left(j_{1}, \ldots, j_{s}\right) \cdots$, and gives the product of the traces

$$
\operatorname{tr}\left(A_{i_{1}} \cdots A_{i_{r}}\right) \operatorname{tr}\left(A_{j_{1}} \cdots A_{j_{s}}\right) \cdots .
$$

It is convenient to introduce the measures

$$
\mu_{\ell}\left(d x_{1} \cdots d x_{\ell}\right)=\operatorname{tr}\left(\prod_{\alpha=1}^{k} \widehat{\sigma} F\left(d x_{\alpha}\right)\right),
$$

so that $\mu_{1}(d x)=\widehat{m}_{1}(d x)$, and (33) reads $\widehat{m}_{1}(d x d y)=\mu_{1}(d x) \mu_{2}(d x)+s \mu_{2}(d x d y)$. Then, for example, for $k=3$, we find

$$
\begin{gathered}
\widehat{m}_{3}(d x d y d z)=\mu_{1}(d x) \mu_{1}(d y) \mu_{1}(d z)+s\left(\mu_{2}(d x d y) \mu_{1}(d z)+\text { cyclic }\right) \\
+2 \Re e \mu_{3}(d x d y d z) .
\end{gathered}
$$

For general $k$ we get similar expansions into products of measures, the combinatorics of which requires some representation theory of the permutation group, which we will not expound here.

\section{D. "Small s" and parastatistics}

We end this section with a remark on parastatistics and weak beams. If we write (29) as

$$
C_{s}(f)=\exp \operatorname{tr}\left(\frac{-1}{s} \log \left(\mathbb{1}-s \widehat{\sigma} F\left[e^{i f}-1\right]\right)\right),
$$

the formula gives corrected Boltzmann statistics (25) for $s=0$, and parafermi (resp. parabose) statistics of order $p$ for $s=-1 / p$ (resp. $s=1 / p)$. From (36) we get a rather uniform notion of weak beams: Whenever $\sigma$ is small (or in the parastatistics case: when $s$ is small), we nearly get Poisson 
statistics. In quantitative terms, the operator norm $\|\widehat{\sigma}\|_{\infty}$ (the largest eigenvalue) measures well the maximal effect of statistics, in some sense the maximal phase space density:

$$
\left|\log C_{s}(f)-\log C_{0}(f)\right| \leq\|\widehat{\sigma}\|_{1} \beta\left(|s|\|\widehat{\sigma}\|_{\infty}\right) \quad \text { with } \beta(h)=-1-\frac{\log (1-h)}{h} \approx \frac{h}{2} .
$$

\section{E. For comparison: Coherent states}

Quasi-free states (which are associated to, e.g., thermal light) are very different from coherent states, which are commonly used in describing laser beams. In order to make the distinction clear, we derive here the characteristic function and factorial moments for the latter case. The (Bose) coherent states are given by the non-normalized vectors

$$
e^{\phi}=\bigoplus_{N=0}^{\infty} \frac{1}{\sqrt{N !}} \phi^{\otimes N},
$$

in the Bose Fock space $\Gamma_{+}(\mathcal{H})$. The characteristic function (15) is now

$$
C(f)=\left\langle e^{\phi \sim} \mid \Gamma_{+}\left(F\left[e^{i f}\right]\right) e^{\phi}\right\rangle /\left\|e^{\phi}\right\|^{2}=\exp \left\langle\phi \mid F\left(e^{i f}-1\right) \phi\right\rangle .
$$

This is precisely the characteristic function (4) of a Poisson random field with intensity measure

$$
\mu(d x)=\langle\phi \mid F(d x) \phi\rangle .
$$

It is remarkable that this holds for any second quantized observable. The factorial moments are now simply products of $\mu$ :

$$
\widehat{m}_{k}\left(d x_{1} \cdots d x_{k}\right)=\mu\left(d x_{1}\right) \cdots \mu\left(d x_{k}\right) .
$$

Comparing with (33), we see that a quasi-free state with the same first moment, i.e., with $\widehat{\sigma}=|\phi\rangle\langle\phi|$, has larger second moment. In particular, thermal light has more variance than coherent light of same intensity.

From (41) we also immediately see that for any coherent state and any counting observable, all the correlation functions (13) are constant, $g^{(n)}\left(x_{1}, \ldots, x_{k}\right)=1$, as expected. ${ }^{6,19}$

\section{LOCAL COUNTING STATISTICS FOR STATIONARY PARTICLE BEAMS}

The main aim of our paper is to establish local counting statistics even in situations where the global particle count is infinite, as will be the case for any stationary beam, or translationally invariant gas. We will start by proving an essential technical result needed for the "localization," and then we present a physically realistic particle creation model leading to a stationary beam in a suitable long time limit. Finally, we put this together with the arrival time measurements to provide concrete formulas (e.g., correlation functions) for the statistics of the "click" times for the particle detection from the beam.

The idea is to use the formula (29) for the characteristic function even in situations, where the operator $\widehat{\sigma}$ has infinite trace, but the product $\widehat{\sigma} F$ is sufficiently well behaved so the formula makes sense as written. Actually, even more general situations can be covered, if we replace $\widehat{\sigma} F$ by $\sqrt{\widehat{\sigma}} F \sqrt{\widehat{\sigma}}$. Indeed, we will show that under a "local trace class condition," which holds in case $F$ is an arrival time measurement and $\widehat{\sigma}$ describes a stationary beam as considered in Sec. IV C below, the characteristic function can be written as

$$
C(f)=\operatorname{det}\left(\mathbb{1}-s \sqrt{\widehat{\sigma}} F\left[e^{i f}-1\right] \sqrt{\widehat{\sigma}}\right)^{-s} .
$$




\section{A. The localization lemma}

The characteristic function (42) is the same as (29) when $\widehat{\sigma}$ has finite trace. Indeed, we have the identity ${ }^{26} \operatorname{det}(\mathbb{1}+A B)=\operatorname{det}(\mathbb{1}+B A)$, whenever both $A B$ and $B A$ are both trace class. In the case at hand this is applied to the two Hilbert-Schmidt operators $A=\sqrt{\widehat{\sigma}}$ and $B=F\left[e^{i f}-1\right] \sqrt{\widehat{\sigma}}$. The form given in the following lemma is even slightly more general.

Lemma 1. Let $F$ be a measure on some set $X$, whose values are positive operators on a Hilbert space $\mathcal{H}_{1}$, with $F(X) \leq \mathbb{1}$, and consider a subset $X_{0} \subset X$. Let $\mathcal{H}_{2}$ be another Hilbert space and $W: \mathcal{H}_{2} \rightarrow \mathcal{H}_{1}$ a bounded operator such that

$$
\operatorname{tr} W^{*} F\left(X_{0}\right) W<\infty
$$

and in the Fermi case $(s=-1)$ also $\|W\|<1$. Then the formula

$$
C(f)=\operatorname{det}\left(\mathbb{1}-s W^{*} F\left[e^{i f}-1\right] W\right)^{-s},
$$

for all $f$ vanishing outside $X_{0}$ defines the characteristic function of a point process in $X_{0}$.

Proof. Consider the Naimark dilation $F=V^{*} \widetilde{F} V$. Then for $f$ with support in $X_{0}$ we can write

$$
W^{*} F[f] W=W^{*} V^{*} \widetilde{F}[f] V W=W^{*} V^{*} \widetilde{F}\left(X_{0}\right) \widetilde{F}[f] \widetilde{F}\left(X_{0}\right) V W=\widetilde{W}^{*} \widetilde{F}[f] \widetilde{W},
$$

where $\widetilde{W}=\widetilde{F}\left(X_{0}\right) V W$, and at the second equality we used the projection valuedness of $\widetilde{F}$. Now by assumption (43) the operator $\widetilde{W}^{*} \widetilde{W}$ has finite trace, i.e., $\widetilde{W}$ is a Hilbert-Schmidt operator. Moreover (relevant only for $s=-1)\|W\|<1$ implies $\|\widetilde{W}\|<1$, because $\widetilde{F}\left(X_{0}\right)$ is a projection, and the dilation operator $V$ satisfies $V^{*} V=F(X) \leq \mathbb{1}$. Hence $\widetilde{W} \widetilde{W}^{*}$ satisfies all conditions required of an operator $\widehat{\sigma}$ to define the counting statistics of a bona fide quasi-free state, with respect to a second quantized observable. The associated characteristic function (29) is

$$
C(f)=\operatorname{det}\left(\mathbb{1}-s \widetilde{W} \widetilde{W}^{*} \widetilde{F}\left[e^{i f}-1\right]\right)^{-s},
$$

which has the form stated in the lemma by the same argument that gave the equality of (29) and (42) at the beginning of this section.

Since we can take $W=\sqrt{\widehat{\sigma}}$ in the lemma, we find $\operatorname{tr} \sqrt{\widehat{\sigma}} F\left(X_{0}\right) \sqrt{\widehat{\sigma}}<\infty$ as a sufficient condition to apply (42). The similar looking condition $\left\|\widehat{\sigma} F\left(X_{0}\right)\right\|_{1}<\infty$, which is suggested by the characteristic function (29), is actually stronger. This is implied by the estimate $\operatorname{tr} \sqrt{A} B \sqrt{A} \leq\|A B\|_{1}$, which holds for arbitrary positive operators $A, B$. (For a proof note that the trace norm is the sum of the singular values, which dominates the sum of the absolute values of the eigenvalues (Theorem 1.15 of Ref. 14), and that $A B$ and $\sqrt{A} B \sqrt{A}$ have the same nonzero eigenvalues.)

As a byproduct we can now approximate the counting statistics of a stationary state by the counting statistics of finite-beam ones:

Lemma 2. Suppose that $W_{0}: \mathcal{H}_{2} \rightarrow \mathcal{H}_{1}$ satisfies the conditions of Lemma 1 for a given measure $F$ and a set $X_{0} \subset X$. Let $W_{\infty}, W_{\alpha}: \mathcal{H}_{2} \rightarrow \mathcal{H}_{1}$ be bounded operators, such that $W_{\alpha}^{*} \rightarrow W_{\infty}^{*}$ strongly, and

$$
W_{\alpha} W_{\alpha}^{*} \leq W_{0} W_{0}^{*}
$$

Then $W_{\infty}$ and each $W_{\alpha}$ satisfy the conditions of Lemma 1, and for the associated characteristic functions $C_{\alpha}, C_{\infty}$ holds

$$
C_{\infty}(f)=\lim _{\alpha} C_{\alpha}(f)
$$

uniformly for $f$ with support in $X_{0}$.

Proof. Defining $\widetilde{W}_{\alpha}$ for $W_{\alpha}$ as in Lemma 1, the assumption (46) gives $\widetilde{W}_{\alpha} \widetilde{W}_{\alpha}^{*} \leq \widetilde{W}_{0} \widetilde{W}_{0}^{*}$, which implies that the conditions of Lemma 1 are valid also for each $W_{\alpha}$ and $W_{\infty}$, and that $\left\|\widetilde{W}_{\alpha}^{*} \psi\right\|^{2}$ $\lesssim\left\|\widetilde{W}_{0}^{*} \psi\right\|^{2}$ for $\psi \in \mathcal{H}$ where now $\widetilde{W}_{0}^{*}$ is Hilbert-Schmidt. Together with the strong convergence $\widetilde{W}_{\alpha}^{*} \rightarrow \widetilde{W}_{\infty}^{*}$, this implies convergence in the Hilbert-Schmidt norm. Hence, $\widetilde{W}_{\alpha} \widetilde{W}_{\alpha}^{*} \rightarrow \widetilde{W}_{\infty} \widetilde{W}_{\infty}^{*}$ in 
the trace norm. Using (45), we get the uniform convergence of the $C_{\alpha}$ from the estimate $\mid \operatorname{det}(\mathbb{1}$ $+A)-\operatorname{det}(\mathbb{1}+B) \mid \leq\|A-B\|_{1} e^{\|A\|_{1}+\|B\|_{1}+1} \cdot 26$

Now, in order to approximate a stationary beam with non-trace class $\widehat{\sigma}$, by a sequence of finite beams with trace class $\widehat{\sigma}_{\alpha}$, it is sufficient to have the weak operator convergence $\sqrt{\widehat{\sigma}}_{\alpha} \rightarrow \sqrt{\widehat{\sigma}}$, and majorization $\widehat{\sigma}_{\alpha} \leq \widehat{\sigma}_{0}$ by some $\widehat{\sigma}_{0}$ with $\operatorname{tr} \sqrt{\widehat{\sigma}_{0}} F\left(X_{0}\right) \sqrt{\widehat{\sigma}_{0}}<\infty$. This will give uniform convergence of characteristic functions for the local counting statistics.

\section{B. Stationary limit of a particle source}

We will now present a concrete way of building a stationary source starting from a particle creation in the Fock space. To get an idea of the result we are looking for, let us briefly go back to the Boltzmann statistical model (25). We assumed there that $\mu$ is a finite measure, so the total number of particles had finite expectation. But we can also take $\mu(d t)=\mu_{0} d t$ as a multiple of Lebesgue measure, where $\mu_{0}$ is the emission rate, i.e.,

$$
\sigma=\mu_{0} \int d t \sigma(t)
$$

provided the integral is a bounded operator and, for finite intervals $S, \operatorname{tr} \sqrt{\sigma} F(S) \sqrt{\sigma}$ has finite trace, as discussed in Subsection IV A. One can take this as a motivation for looking integrated trace class operators (47) also in the case of Bose/Fermi statistics. We will now show how to arrive at such an operator from a physically realistic description of beam generation which is consistent with statistics from the outset.

For building a stationary source model it is best to include the particle generation in the dynamics. In this way one can consider sources operating continuously for an arbitrarily long time. The intuitive idea is that after being activated at time $t=0$, the source creates particles with fixed initial wave function $\phi$, one after another, each particle subsequently evolving according to some single particle Hamiltonian $H$ with direct integral decomposition as discussed in Sec. II C. Formally, this is expressed (see, e.g., the review, ${ }^{27,28}$ ) by evolving the many particle state $\rho_{t}$ according to the master equation

$$
\frac{d}{d t} \rho_{t}=-i\left[d \Gamma(H), \rho_{t}\right]+\lambda\left(2 a_{\phi} \rho_{t} a_{\phi}^{\dagger}-a_{\phi} a_{\phi}^{\dagger} \rho_{t}-\rho_{t} a_{\phi} a_{\phi}^{\dagger}\right),
$$

with the initial condition that $\rho_{0}$ is the vacuum state. Here and in the rest of this section we consider only the Bosonic case. Then $\lambda>0$ quantifies the strength of the source, and $d \Gamma(H)$ is the many particle Hamiltonian corresponding to $H$. The time evolution $t \mapsto \rho_{t}$ is a quasi-free semigroup, ${ }^{27}$ so the Fock space state $\rho_{t}$ is quasi-free for each $t \geq 0$. This reduces the dynamics to a one-particle problem, which has the solution

$$
\widehat{\sigma}_{\phi}(t)=2 \lambda \int_{0}^{t} d s e^{s T_{\phi}}|\phi\rangle\langle\phi|\left(e^{s T_{\phi}}\right)^{*},
$$

where $T_{\phi}=-i H+\lambda|\phi\rangle\langle\phi|$ is the generator of a strongly continuous semigroup.

The integral $\widehat{\sigma}_{\phi}(\infty)$ is the analogue of (47). To compute it, we can formally solve the function $\beta_{\psi}(t)=\Theta(t)\left\langle e^{t T_{\phi}} \phi \mid \psi\right\rangle$, in terms of $\gamma_{\psi}(t)=\Theta(t)\left\langle e^{-i t H} \phi \mid \psi\right\rangle$, where $\Theta$ is the Heaviside step function. In fact, we get $\breve{\beta}_{\psi}=S_{\phi} \check{\gamma}_{\psi}$, where $\breve{h}$ denotes the inverse of the Fourier transform given in (24), and

$$
S_{\phi}(E)=\left(1-\lambda \check{\gamma}_{\phi}(E)\right)^{-1} \text {. }
$$

This gives $\left\langle\psi \mid \widehat{\sigma}_{\phi}(\infty) \psi\right\rangle=\lambda \pi^{-1} \int_{-\infty}^{\infty}\left|S_{\phi}(E) \check{\gamma}_{\psi}(E)\right|^{2} d E$, corresponding to a quasi-free state which is invariant with respect to the evolution (48) but not with respect to the free Hamiltonian $H$.

In order to get a state which is stationary for the free evolution, we now take another limit $\widehat{\sigma}=\lim _{s \rightarrow+\infty} e^{i H s} \widehat{\sigma}_{\phi}(\infty) e^{-i H s}$. In the spirit of scattering theory, this amounts to translating the state back in time with the free evolution, after having let it evolve a long time according to the particle generating semigroup evolution. Since $\gamma_{e^{-i H s} \psi}(t)=\gamma_{\psi}(t-s)$, we have $e^{i s E} \breve{\gamma}_{e^{-i H s} \psi}(E)$ $\rightarrow 2 \pi\langle\phi(E) \mid \psi(E)\rangle$ as $s \rightarrow+\infty$, where $\phi(E)$ is the wave function $\phi$ in the $H$ (energy) representation 
(20). Here we have used the fact that

$$
\left\langle e^{-i t H} \phi \mid \psi\right\rangle=\int e^{i t E}\langle\phi(E) \mid \psi(E)\rangle_{\mathcal{H}_{E}} .
$$

Thus the final stationary limit is given by

$$
\widehat{\sigma}=4 \pi \lambda \int^{\oplus} d E\left|S_{\phi}(E)\right|^{2}\left|\phi_{E}\right\rangle\left\langle\phi_{E}\right| .
$$

In the $1 \mathrm{D}$ case with the free Hamiltonian, one can alternatively take a limit of moving the source to $-\infty$ in space, which results in a similar expression but with the projection onto positive momenta. Even in this case the denominator, which reflects the phase space density at the source, still depends on the negative momentum components of $\phi$.

There are, of course, some assumptions needed to make the above derivation valid. Physically, we expect ${ }^{28}$ that the free evolution $H$ should be fast enough compared to the strength of the source so that the particles do not accumulate, or even condense, near the source, but move away as new ones are created. Mathematically, the relevant assumptions can be expressed as follows: (i) The wave function $\phi$ is bounded in the energy representation and (ii) the operator of multiplication by $S_{\phi}(E)$ defines a bounded operator which keeps the Hardy class $H^{2}$ - invariant. The assumption (i) ensures that $\left\|\gamma_{\psi}\right\|_{2} /\|\psi\|$ is uniformly bounded due to (51); in particular, the $L^{2}$ Fourier transform $\check{\gamma}_{\phi}$ belongs to the Hardy class $H^{2}$, and so can be extended to an analytic function in the open lower half plane. Then (ii) implies that $S_{\phi} \check{\gamma}_{\psi}$ is square integrable, and has support on $[0, \infty)$; hence $S_{\phi}$ is well defined, the formal relation $\breve{\beta}_{\psi}=S_{\phi} \check{\gamma}_{\psi}$ makes sense, and (52) is bounded, the limits existing in the weak operator topology.

The assumption (ii) can be replaced with stronger but more easily verifiable versions: for instance, if $\gamma_{\phi}$ is (absolutely) integrable, and $\check{\gamma}_{\phi}(E) \neq \lambda^{-1}$ for all $E$ in the closed lower half plane, then (ii) holds. Even stronger condition ${ }^{28}$ is $\lambda \int_{0}^{\infty}\left|\gamma_{\phi}(t)\right| d t<1$.

The assumption (ii) implies, in particular, that $\int\left|S_{\phi}(E)\right|^{2}\left\|\phi_{E}\right\|^{2} d E<\infty$. In the next section we will see in a more general context that this condition ensures $\operatorname{tr} \sqrt{\widehat{\sigma}} F[f] \sqrt{\widehat{\sigma}}<\infty$ for any arrival time observable $F$ as in Sec. II C, and any $f$ compactly supported in the time direction. By Lemma 1 , the local counting statistics of $\Gamma_{+} F$ is therefore well-defined for the stationary state (52), and can be obtained from (42).

In order to complete the discussion on this stationary limit, we have to check that the counting statistics for the limit state (52) can be approximated by measuring the actual finite beam emitted by the source. It is clear by construction that we can write $\widehat{\sigma}_{s, t}=e^{i H s} \widehat{\sigma}_{\phi}(t) e^{-i H s}=W_{s, t}^{*} W_{s, t}$, where $\left(W_{s, t} \psi\right)(E)=\sqrt{\lambda / \pi} e^{i s E} \int_{0}^{t} e^{-i E t^{\prime}} \beta_{\phi, e^{-i s H} \psi}\left(t^{\prime}\right) d t^{\prime}$. Here $W_{s, t}$ converge strongly when we take first $t \rightarrow \infty$ and then $s \rightarrow \infty$. Moreover, $\widehat{\sigma}_{s, t} \leq\left\|S_{\phi}\right\|^{2} \widehat{\sigma}_{0}$, where $\widehat{\sigma}_{0}$ is (52) without $\left|S_{\phi}(E)\right|^{2}$. Hence, it follows from Lemma 2 that

$$
\lim _{s \rightarrow \infty} \lim _{t \rightarrow \infty} C_{s, t}(f)=\operatorname{det}\left(\mathbb{1}-\sqrt{\widehat{\sigma}} F\left[e^{i f}-1\right] \sqrt{\widehat{\sigma}}\right)^{-1},
$$

where $C_{s, t}$ is the characteristic function (42) corresponding to the operator $\widehat{\sigma}_{s, t}$.

\section{General time-stationary beams}

The following general scheme emerges from the above. We consider systems with Hamiltonian $H$, and decompose the Hilbert space into a direct integral $\mathcal{H}=\int{ }^{\oplus} d E \mathcal{H}_{E}$ over the spectrum of $H$. Stationary beams are described by an extension of quasi-free states, given in terms of a one-particle operator

$$
\widehat{\sigma}=\int^{\oplus} d E \widehat{\sigma}(E),
$$

commuting with the Hamiltonian. Here $\widehat{\sigma}(E)$ is a positive trace class operator in the multiplicity space $\mathcal{H}_{E}$ at $E$, which depends on the details of the source. The basic normalization condition for 
these operators is that

$$
\gamma=\frac{1}{2 \pi} \int d E \operatorname{tr} \widehat{\sigma}(E)<\infty .
$$

We emphasize that no such operator is trace class. Indeed, a general trace class operator $T$ would have an integral kernel $T\left(E, E^{\prime}\right): \mathcal{H}_{E^{\prime}} \rightarrow \mathcal{H}_{E}$ so that $(T \psi)(E)=\int d E^{\prime} T\left(E, E^{\prime}\right) \psi\left(E^{\prime}\right)$. The trace of such an operator is $\operatorname{tr} T=\int d E \operatorname{tr} T(E, E)$. In contrast, the operator (54) has the formal integral kernel $T\left(E, E^{\prime}\right)=\widehat{\sigma}(E) \delta\left(E-E^{\prime}\right)$, which is singular on the diagonal. Such operators do arise from integration of trace class operators over time in the sense of (47): The time evolved operator $U_{t}^{*} T U_{t}$ has integral kernel $T\left(E, E^{\prime}\right) \exp \left(i t\left(E-E^{\prime}\right)\right)$. Integrating this with respect to time we get the kernel $2 \pi \delta\left(E-E^{\prime}\right) T(E, E)$. Hence the trace class condition for $T$ turns into (55) for the integral $\int d t U_{t}^{*} T U_{t}$.

The direct integral form (54) or, in other words, the elimination of off-energy-diagonal terms in the kernel for $\widehat{\sigma}$ is, of course, just the consequence of stationarity $[\widehat{\sigma}, H]=0$, and leads to a major simplification in the computation of expectation values and rates.

\section{Time-local counting statistics}

We now want to combine the stationary sources given by $\widehat{\sigma}$ of the form (54), with a general counting observable $\Gamma_{s} F$, i.e., the second quantization of a general arrival time observable $F$, as discussed in Sec. II C. The full local counting statistics is then contained in the characteristic function $C(f)$ restricted to test functions $f$ which have compact support in the time direction. Technically, this approach is based on the discussion in Sec. IV A, which guarantees the existence of $C(f)$ in $(42)$ for any $f$ with $f(x, t)=0$ for $t \notin\left[t_{1}, t_{2}\right]$, once we have the "local trace class condition"

$$
\operatorname{tr} \sqrt{\widehat{\sigma}} F[\chi] \sqrt{\widehat{\sigma}}<\infty
$$

where $\chi(x, t)=1$ for $t \in\left[t_{1}, t_{2}\right]$ and $\chi(x, t)=0$ otherwise. One of the consequences of this approach is that we can always get a justification of the formula starting from finitely extended beams (trace class $\widehat{\sigma}$ ) and going to a stationary limit in (42). There are many ways to do such a limit, which corresponds to the many ways a beam which looks basically stationary during a fixed time interval could begin in the distant past and end in the far future. The formula (42) thus captures the essence of what we mean by "stationary beams."

The rest of this subsection will be devoted to substantiating the above claim (56) for $\widehat{\sigma}$ of the form (54), any time-covariant $F$, and any $t_{1}<t_{2}$. We do this by showing the more general estimate

$$
\|\sqrt{\widehat{\sigma}} F[f] \sqrt{\widehat{\sigma}}\|_{1} \leq\|f\|_{\infty} \gamma\left|t_{2}-t_{1}\right|,
$$

for any bounded complex (measurable) test function $f$ such that $f(t, x)=0$ for $t \notin\left[t_{1}, t_{2}\right]$, where $\gamma$ is the rate constant from (55). First we apply the triangle inequality to a sum of positive operators, and use that on positive elements the trace norm is just the trace. That is for positive operators $F_{\alpha}$ and $f_{\alpha} \in \mathbb{C}$ we have

$$
\left\|\sum_{\alpha} f_{\alpha} F_{\alpha}\right\|_{1} \leq \max _{\alpha}\left|f_{\alpha}\right| \sum_{\alpha} \operatorname{tr} F_{\alpha} .
$$

Hence, for step functions $f=\sum_{\alpha} f_{\alpha} \chi_{\alpha}$, with $\chi=\sum_{\alpha} \chi_{\alpha}$, the left-hand side of (57) is bounded by $\|f\|_{\infty} \operatorname{tr} \sqrt{\widehat{\sigma}} F[\chi] \sqrt{\widehat{\sigma}}$. To prove (57), it is therefore sufficient to show that

$$
\operatorname{tr} \sqrt{\widehat{\sigma}} F[\chi] \sqrt{\widehat{\sigma}} \leq \gamma\left|t_{2}-t_{1}\right| \text {. }
$$

We do this by expressing $F$ by its dilation $F=V^{*} \widetilde{F} V$. Since $\widetilde{F}[\chi]$ is a projection, the trace we need to compute is $\operatorname{tr} W^{*} W$ with

$$
W=\widetilde{F}[\chi] V \sqrt{\widehat{\sigma}} .
$$


Since $\operatorname{tr} W^{*} W=\operatorname{tr} W W^{*}$ for any operator (where both sides might still be infinite), we now compute $\operatorname{tr} W W^{*}=\widetilde{F}[\chi] V \widehat{\sigma} V^{*} \widetilde{F}[\chi]$. Note that

$$
V \widehat{\sigma} V^{*}=\int^{\oplus} d E V_{E} \widehat{\sigma}(E) V_{E}^{*}
$$

commutes with the energy. Therefore, in the time domain it acts as a convolution operator:

$$
\begin{aligned}
\left(V \widehat{\sigma} V^{*} \Phi\right)(t) & =\int d s S(t-s) \psi(s) \\
\text { with } \quad S(t) & =\frac{1}{2 \pi} \int d E e^{i t E} V_{E} \widehat{\sigma}(E) V_{E}^{*} .
\end{aligned}
$$

Here the integral defining $S$ is convergent in trace norm by assumption (55), and by the RiemannLebesgue lemma $t \mapsto S(t) \in \mathcal{B}(\mathcal{K})$ is a continuous function vanishing at infinity. Due to the continuity we can evaluate the trace as an integral on the diagonal of the kernel $K(t, s)=S(t-s)$ (Theorem 3.9 of Ref. 14):

$$
\operatorname{tr} \widetilde{F}[\chi] V \widehat{\sigma} V^{*} \widetilde{F}[\chi]=\int_{t_{1}}^{t_{2}} d t \operatorname{tr}_{\mathcal{K}} S(0)
$$

But

$$
\operatorname{tr}_{\mathcal{K}} S(0)=\frac{1}{2 \pi} \int d E \operatorname{tr} \widehat{\sigma}(E) V_{E}^{*} V_{E} \leq \gamma .
$$

This completes the proof of the estimate (57).

According to Sec. IV A, in order to approximate the counting statistics of a stationary state (described by $\widehat{\sigma}$ of the form (54)) by the counting statistics of finite-beam ones (with trace class $\widehat{\sigma}_{\alpha}$ ), it is sufficient to find one majorizing $\widehat{\sigma}_{0}$ of the form (54), with $\widehat{\sigma}_{\alpha} \leq \widehat{\sigma}_{0}$, and have $\sqrt{\widehat{\sigma}_{\alpha}} \rightarrow \sqrt{\widehat{\sigma}}$ at least weakly. This will give uniform convergence of characteristic functions for the local counting statistics.

\section{E. Rates and correlation functions}

Now that we have established well-defined counting statistics for the stationary beams considered above, we can extract the counting rates and correlation functions from the associated characteristic function. For this we need the moments, which are best expressed in terms of the operator valued function $S(t)$, defined in (62). This depends both on the source via $\widehat{\sigma}$ and on the observable chosen, via $V$. It also contains the required Fourier transformations, so all moments are immediately expressed in the time domain. The idea is to reduce the factorial moments to the measures from (34), rewritten by using the dilation:

$$
\mu_{\ell}\left(d x_{1} \cdots d x_{\ell}\right)=\operatorname{tr}\left(V \widehat{\sigma} V^{*} \widetilde{F}\left(d x_{1}\right) \cdots V \widehat{\sigma} V^{*} \widetilde{F}\left(d x_{\ell}\right)\right) .
$$

The operator under the trace has integral kernel

$$
K\left(t_{0}, t_{\ell}\right)=\int d t_{1} \cdots d t_{\ell-1} S\left(t_{0}-t_{1}\right) \widetilde{F}\left(d x_{1}\right) S\left(t_{1}-t_{2}\right) \cdots S\left(t_{\ell-1}-t_{\ell}\right) \widetilde{F}\left(d x_{\ell}\right) .
$$

Then the required trace is $\int d t \operatorname{tr} K(t, t)$, where the trace in the integrand is over $\mathcal{K}$. In all these expressions the argument of the measure $\widetilde{F}$ is still the combination of time and arrival location. But noting that with respect to time $\widetilde{F}$ is just a multiplication operator, so for $f(t, x)=h(t) g(x)$ we have $(\widetilde{F}[h g] \Phi)(t)=h(t) G[g] \Phi(t)$. Here and in the following $x$ stands only for the arrival location. If we now take $h$ and $g$ as the indicator functions of small sets $d t \subset \mathbb{R}$ and $d x \subset X$, we find that

$$
\mu_{\ell}\left(d t_{1} d x_{1}, \cdots, d t_{\ell} d x_{\ell}\right)=d t_{1} \cdots d t_{\ell} \operatorname{tr}\left(S\left(t_{\ell}-t_{1}\right) G\left(d x_{1}\right) S\left(t_{1}-t_{2}\right) \cdots G\left(d x_{\ell}\right)\right) .
$$

For the first (factorial) moment we thus get

$$
\widehat{m}_{1}(d t d x)=d t \operatorname{tr} S(0) G(d x) .
$$


Hence $S(0)$ serves as a "density matrix" for count rates. It is normalized to the total particle rate $\gamma$. For a discrete family of counters with POVM elements $G_{x} \geq 0, \sum_{x} G_{x}=\mathbb{1}$ the arrival rate at counter $x$ is $\gamma_{x}=\operatorname{tr} S(0) G_{x}$.

The second moment has a density depending only on the time difference $\tau=t_{2}-t_{1}$. For discrete counters we also give the form of the normalized correlation function $g^{(2)}$ :

$$
\begin{aligned}
\widehat{m}_{2}\left(d t_{1} d x_{1}, d t_{2} d x_{2}\right)= & d t_{1} d t_{2} M_{t_{1}-t_{2}}\left(d x_{1}, d x_{2}\right) \\
M_{\tau}\left(d x_{1}, d x_{2}\right)=\left(\operatorname{tr} S(0) G\left(d x_{1}\right)\right)\left(\operatorname{tr} S(0) G\left(d x_{2}\right)\right) & +s \operatorname{tr} S(\tau)^{*} G\left(d x_{1}\right) S(\tau) G\left(d x_{2}\right) \\
g_{x y}^{(2)}(\tau)= & 1+\frac{s}{\gamma_{x} \gamma_{y}} \operatorname{tr} S(\tau)^{*} G_{x} S(\tau) G_{y} .
\end{aligned}
$$

Here we used the symmetry $S(-\tau)=S(\tau)^{*}$ to write the expression in a more obviously positive form.

It is not very enlightening to write down the higher moments. The third moment (35) will contain contributions $\operatorname{tr} S\left(t_{3}-t_{1}\right) G_{x} S\left(t_{1}-t_{2}\right) G_{y} S\left(t_{2}-t_{3}\right) G_{z}$.

\section{F. Stationarity in time and space}

In this paper we have considered quasi-free beams stationary in time, i.e., those for which $[\widehat{\sigma}, H]=0$, and which consequently are of the form (54). Here we wish to briefly remark on the extension of the formalism to encompass beams stationary also in space. Even for the free Hamiltonian $H=P^{2} / 2$ in one dimension (with $P$ the momentum), $[\widehat{\sigma}, H]=0$ does not imply stationarity in space, $[\widehat{\sigma}, P]=0$. In fact, the beam (52) produced by our source model is not stationary in space if the source state $\phi$ has both positive and negative momentum components. Note that such a situation cannot happen classically.

In order to get beams stationary in both time and space, we would have to replace (54) by an operator diagonal in both $P$ and $H$. In one dimension, this just means that we can equivalently decompose $\widehat{\sigma}$ in the spectral representation of $P$. For the free Hamiltonian in three dimensions, we can take

$$
\widehat{\sigma}=\int^{\oplus} d E d p_{1} d p_{2} \widehat{\sigma}\left(E, p_{1}, p_{2}\right),
$$

if we assume that $\widehat{\sigma}$ is supported in the subspace of positive $P_{3}$, so that we can use $E$ instead of $p_{3}$. In the detector side, we then need screen observables ${ }^{21}$ with outcomes $\left(t, x_{1}, x_{2}, y\right)$ where $t$ is the detection time, $\left(x_{1}, x_{2}\right)$ is the location on the detector screen situated in the $x_{1} x_{2}$-plane, and $y$ is some additional parameter. These observables are by definition covariant for time translations (generated by $H$ ) as well as translations on the screen (generated by $P_{1}$ and $P_{2}$ ), and consequently, the above scheme for computing rates and correlation functions works in a similar fashion also in this case. The function in the local trace class condition must now be compactly supported in both $t$ and $\left(x_{1}\right.$, $x_{2}$ ), corresponding to counting statistics for detections in a given bounded region of the screen (the physical detector), occurring within a given finite time interval.

\section{EXAMPLES}

\section{A. Second order correlation function}

In the case of the free Hamiltonian in one dimension, we have $\mathcal{H}_{E}=\mathbb{C}^{2}$. Taking the source (52), together with the simplest arrival time measurement, i.e., the one with $V_{E}=\mathbb{1}$ for $E \geq 0$ and one detector $G$ corresponding to the detection of right going (positive momenta) particles, we get

$$
\gamma=(2 \pi)^{-1} \chi(0), \quad g^{(2)}(\tau)=1+|\chi(\tau)|^{2} \chi(0)^{-2},
$$

where $\chi(\tau)=4 \pi \lambda \int_{0}^{\infty} d E e^{-i \tau E}\left|\phi_{E,+}\right|^{2}|1-\lambda h(E)|^{-2}$, and $\phi_{E,+}$ is the positive momentum component of $\phi_{E}$. In Fig. $2, g^{(2)}(\tau)$ is shown for a Lorentzian $\left|\phi_{E,+}\right|^{2} \sim(\alpha / 2) /\left(\left(E-E_{0}\right)^{2}+(\alpha / 2)^{2}\right) / \pi$ and a 


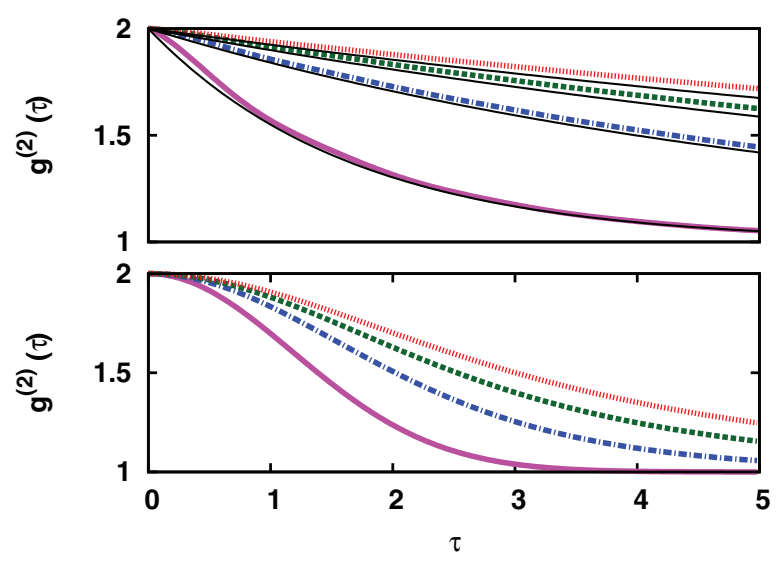

FIG. 2. Second order normalized correlation function $g^{(2)}(\tau)$. Upper figure: Lorentzian, lower figure: Gaussian initial spectral density. Boltzmann model (thick solid line), quasi-free state given by (52) with rates $\gamma=0.5,1.0,1.5$ (bottom to top curves).

Gaussian $\left|\phi_{E,+}\right|^{2} \sim \exp \left(-\left(E-E_{0}\right)^{2} /\left(2 \alpha^{2}\right)\right) /(\sqrt{2 \pi} \alpha)$, with no negative momentum components. In the case of the Lorentzian the correlation function can be approximated for $E_{0} \gg \alpha$ by $g^{(2)} \approx 1$ $+e^{-\tau(\alpha-2 \lambda)}$ (see the solid lines in Fig. 2), i.e., as an exponential modified by the intensity parameter $\lambda$, as Bose statistical effects become more relevant.

\section{B. Plane wave beams}

The energy density of the beam is $\operatorname{tr} \widehat{\sigma}(E)$, which clearly needs to be integrable. In the Fermi case the constraint $\widehat{\sigma} \leq \mathbb{1}$ excludes singularities in this density. However, in the Bose case, we can also consider singular distributions. Let us assume a one-dimensional, free Hamiltonian and we restrict to only positive momenta to simplify the notation. In this case the beam state is given by

$$
\hat{\sigma}=\int_{0}^{\infty} d E \alpha(E)|E\rangle\langle E|,
$$

where $|E\rangle$ are the generalized energy eigenvectors. Consider a sequence of functions $\alpha_{n}$ with $\alpha_{n}(E) \stackrel{n \rightarrow \infty}{\longrightarrow} \kappa \delta\left(E-E_{0}\right)=: \alpha(E)$. The characteristic function in this case is

$$
C(f)=\left(1-\kappa\left\langle E_{0}\left|F\left[e^{i f}-1\right]\right| E_{0}\right\rangle\right)^{-1} .
$$

The rate is then given by $\gamma_{Q}=\kappa\left\langle E_{0}|F[f]| E_{0}\right\rangle$. We can get the number distributions $p_{n}$ for a measurement result in an interval $Y$ from the characteristic function, see (10). This characteristic function for $\alpha(E)=\kappa \delta\left(E-E_{0}\right)$ is

$$
C\left(\lambda \chi_{Y}\right)=\frac{1}{1-\left(e^{i \lambda}-1\right) \kappa\left\langle E_{0}|F(Y)| E_{0}\right\rangle}=\sum_{n=0}^{\infty} \frac{q^{n}}{(1+q)^{n+1}} e^{i \lambda n},
$$

where $q=\kappa\left\langle E_{0}|F(I)| E_{0}\right\rangle$. By comparing (67) and (10), we get the number distribution for a detection in the interval $Y$, namely, $p_{Q, n}=\frac{q^{n}}{(1+q)^{n+1}}$. Figure 3 shows examples of the number distribution for an arrival-time measurement with $q=\sqrt{10}$.

It is illustrative to compare it with the number statistics of a coherent beam given by (39) with $\phi(E)=\sqrt{\kappa} \delta\left(E-E_{0}\right)$. The characteristic function is then

$$
C(f)=\exp \left(\kappa\left\langle E_{0}\left|F\left[e^{i f}-1\right]\right| E_{0}\right\rangle\right)
$$

and from this we get the rate $\gamma_{C}=\kappa\left\langle E_{0}|F[f]| E_{0}\right\rangle=\gamma_{Q}$ and a Poisson number distribution $p_{C, n}$ $=\frac{1}{n !} q^{n} e^{-q}$ which is also shown in Fig. 3 . 


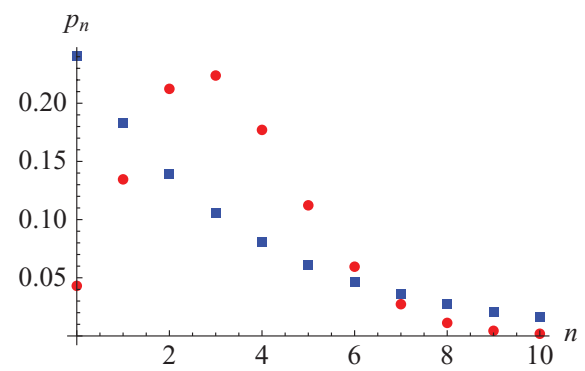

FIG. 3. Particle number distribution $p_{n}$ with $q=\sqrt{10}$, quasi-free beam $p_{Q, n}$ (blue boxes), coherent beam $p_{C, n}$ (red circles).

Let us now again take the specific arrival time measurement with $V_{E}=\mathbb{1}$. In that case, we get the rate $\gamma_{K}=\frac{\kappa}{2 \pi}$ and $q_{K}=\gamma_{K} l(Y)$ where $l(Y)=\int_{Y} d t$. For a quasi-free beam we get for the probability for no detection in an interval $\left[t_{1}, t_{2}\right]$ is $p_{Q, 0}\left(t_{1}, t_{2}\right)=1 /\left(1+\gamma_{K}\left(t_{2}-t_{1}\right)\right)$ and for a coherent beam $p_{C, 0}=e^{-\gamma_{K}\left(t_{2}, t_{1}\right) q}$. The waiting time calculated by (12) is now for a quasi-free beam

$$
w_{Q}(\tau)=\frac{2 \gamma_{K}}{\left(1+\gamma_{K} \tau\right)^{3}}
$$

and for a coherent beam

$$
w_{C}(\tau)=\gamma_{K} e^{-\gamma_{K} \tau},
$$

which is - as expected - an exponential distribution.

\section{ACKNOWLEDGMENTS}

The authors acknowledge support by the BMBF (Ephquam project), the EU project CORNER, and the Academy of Finland.

${ }^{1}$ K. Mølmer, “Optical coherence: A convenient fiction,” Phys. Rev. A 55, 3195-3203 (1997).

${ }^{2}$ S. J. van Enk and C. A. Fuchs, "Quantum state of an ideal propagating laser field," Phys. Rev. Lett. 88, 027902 (2001).

${ }^{3}$ H. J. Carmichael and C. Noh, "Open system entanglement and the laser quantum state," Physica E 42, 399-406 (2010).

${ }^{4}$ A. Lamas-Linares and C. Kurtsiefer, "Breaking a quantum key distribution system through a timing side channel," Opt. Express 15, 9388-9393 (2007).

${ }^{5}$ J.-Å. Larsson and R. D. Gill, "Bell's inequality and the coincidence-time loophole," Europhys. Lett. 67, 707 (2004).

${ }^{6}$ D. F. Walls and G. J. Milburn, Quantum Optics (Springer-Verlag, Berlin, 1994).

${ }^{7}$ Z. Yuan, B. E. Kardynal, R. M. Stevenson, A. J. Shields, C. J. Lobo, K. Cooper, N. S. Beattie, D. A. Ritchie, and M. Pepper, Science 295, 102-105 (2002).

${ }^{8}$ D. Daley and D. Vere-Jones, An Introduction to the Theory of Point Processes (2 vols.) (Springer, 1988).

${ }^{9}$ K. R. Parthasarathy, An Introduction to Quantum Stochastic Calculus (Birkhäuser, 1992).

${ }^{10}$ F. Verstraete and J. Cirac, "Continuous matrix product states for quantum fields," Phys. Rev. Lett. 104, 190405 (2010).

${ }^{11}$ T. J. Osborne, J. Eisert, and F. Verstraete, "Holographic quantum states," Phys. Rev. Lett. 105, 260401 (2010).

${ }^{12}$ C. Bénard, "Fluctuations of beams of quantum particles," Phys. Rev. A 2, 2140-2153 (1970).

${ }^{13}$ C. Bénard and O. Macchi, "Detection and 'emission' processes of quantum particles in a 'chaotic state', J. Math. Phys. 14, 155-167 (1973).

${ }^{14}$ B. Simon, Trace Ideals and Their Applications, 2nd ed. (American Mathematical Society, Providence, RI, 2005).

${ }^{15}$ A. Soshnikov, "Determinantal random point fields," Russ. Math. Surveys 55, 923-975 (2000).

${ }^{16}$ K.-H. Fichtner and W. Freudenberg, "Point processes and the position distribution of infinite boson systems," J. Stat. Phys. 47, 959-978 (1987).

${ }^{17}$ E. Lytvynov, "Fermion and boson random point processes as particle distributions of infinite free Fermi and Bose gases of finite density," Rev. Math. Phys. 14, 1073-1098 (2002).

${ }^{18}$ R. F. Werner, "Inequalities expressing the Pauli principle for generalized observables," Mathematical Methods in Statistical Mechanics (Leuven University Press, 1989), pp. 179-196.

${ }^{19}$ R. J. Glauber, Quantum Theory of Optical Coherence, Selected Papers and Lectures (Wiley-VCH, Weinheim, 2007).

${ }^{20}$ J. Kijowski, "On the time operator in quantum mechanics and the Heisenberg uncertainty relation for energy and time," Rep. Math. Phys. 6, 361 (1974).

${ }^{21}$ R. F. Werner, "Screen observables in relativistic and nonrelativistic quantum mechanics," J. Math. Phys. 27, 793-803 (1986).

${ }^{22}$ G. W. Mackey, "Imprimitivity for representations of locally compact groups i," Proc. Natl. Acad. Sci. U.S.A. 35, 537-545 (1949). 
${ }^{23}$ R. V. Kadison and J. R. Ringrose, Fundamentals of the Theory of Operator Algebras (Academic Press, 1986), Vol. II.

${ }^{24}$ O. Bratteli and D. W. Robinson, Operator Algebras and Quantum Statistical Mechanics II (Springer, Berlin, 1997).

${ }^{25}$ J. Avron, S. Bachmann, G. Graf, and I. Klich, "Fredholm determinants and the statistics of charge transport," Commun. Math. Phys. 280, 807-829 (2008).

${ }^{26}$ M. Reed and B. Simon, Methods of Modern Mathematical Physics IV: Analysis of Operators (Academic Press, New York, 1972), Chap. XIII.17.

${ }^{27}$ R. Alicki, General Theory and Applications to Unstable Particles, Lecture Notes in Physics Vol. 717 (Springer, Berlin, 2007), pp. 1-46.

${ }^{28}$ M. Butz and H. Spohn, "Dynamical phase transition for a quantum particle source," Ann. Henri Poincare 10, 1223-1249 (2010). 